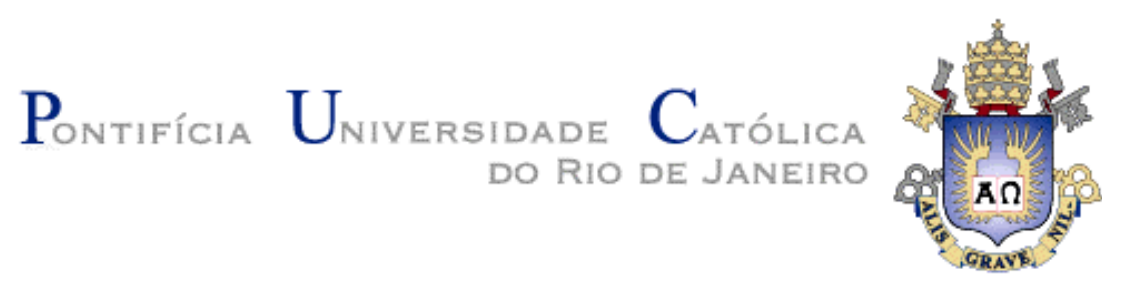

Julio César Bizarreta Ortega

\begin{abstract}
Avaliação do potencial de uso de um resíduo da indústria de papel em sistemas de barreiras capilares
\end{abstract}

Dissertação de Mestrado

Dissertação apresentada como requisito parcial para obtenção do grau de Mestre pelo Programa de Pós-Graduação em Engenharia Civil do Departamento de Engenharia Civil da PUC-Rio.

Orientador: Prof. Tácio Mauro Pereira de Campos

Rio de Janeiro

Agosto de 2009 


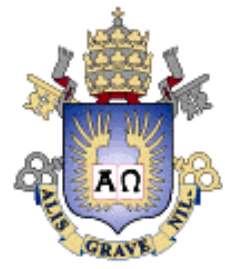

Julio César Bizarreta Ortega

\section{Avaliação do potencial de uso de um resíduo da indústria de papel em sistemas de barreiras capilares}

Dissertação apresentada como requisito parcial para obtenção do título de Mestre pelo Programa de Pós-Graduação em Engenharia Civil da PUCRio. Aprovada pela Comissão Examinadora abaixo assinada.

Prof. Tácio Mauro Pereira de Campos

Presidente / Orientador

Departamento de Engenharia Civil - PUC-Rio

Prof. Alexander Rodrigues Cabral Universite de Sherbrooke (Canadá)

Prof. Fernando Antônio Medeiros Marinho EP-USP

Prof. Eurípides do Amaral Vargas Júnior Departamento de Engenharia Civil - PUC-Rio

Dra. Monica Priscilla Hernandez Moncada Departamento de Engenharia Civil - PUC-Rio

Prof. José Eugênio Leal Coordenador Setorial do Centro Técnico Cientifico- PUC-Rio

Rio de Janeiro, 14 de Agosto de 2009 
Todos os direitos reservados. É proibida a reprodução total ou parcial do trabalho sem autorização da universidade, do autor e do orientador.

\section{Julio César Bizarreta Ortega}

Graduou-se em Engenharia Civil pela Universidad Nacional de Ingenieria (UNI) no Peru em 2002. Ingressou em 2007 no curso de mestrado em Engenharia Civil da PUC-Rio, na área de Geotecnia, e linha de pesquisa de geotecnia ambiental.

Ficha Catalográfica

\section{Bizarreta Ortega, Julio César}

Avaliação do potencial de uso de um resíduo da indústria de papel em sistemas de barreiras capilares / Julio César Bizarreta Ortega; orientador: Tácio Mauro Pereira de Campos - 2009.

108 f. : il ; (color) ;30 cm.

1. Dissertação (Mestrado em Engenharia Civil) Pontifícia Universidade Católica do Rio de Janeiro, Rio de Janeiro, 2009.

Inclui bibliografia.

1. Engenharia civil - Teses. 2. Resíduo da indústria de papel. 3. Caracterização de resíduos. 4. Sistemas de barreiras capilares. 5. Cobertura final. 6. Aterro sanitário I. Campos, Tácio Mauro Pereira de. II. Pontifícia Universidade Católica do Rio de Janeiro. Departamento de Engenharia Civil. III. Título. 


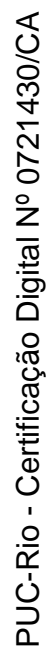

A meus pais, Julio Bizarreta e Angélica Ortega. 


\section{Agradecimentos}

A CAPES e PRONEX pelo financiamento desta dissertação.

Ao Prof. Tácio Mauro Pereira de Campos pela orientação e ensino durante a dissertação.

Ao Wagner Nahas, André Guedes, Jackeline Huertas, Gerson Alves e Julicene Sousa pelas correções feitas nesta dissertação.

Ao Eng. William, Josué e Amauri pelo apoio no laboratório de Geotecnia e Meio Ambiente da PUC.

Ao laboratório de MEV e Raios X do DEMa e ao laboratório de Catálise do Departamento de Química da PUC-Rio.

Aos Professores Franklin Antunes, Roberto de Avillez, Maria Isabel P. da Silva, Sidnei Paciornik, José D' Abreu, Claudia Teixeira, Alexander Cabral pelo apoio às consultas sobre a caracterização do material estudado.

Ao Ivan Benítez Hipólito pelo apoio na aprendizagem do programa VADOSE/W 2007.

Aos professores que participaram da Comissão Examinadora pelas sugestões feitas. 


\section{Resumo}

Bizarreta, Julio César Ortega; de Campos, Tácio Mauro Pereira. Avaliação do potencial de uso de um resíduo da indústria de papel em sistemas de barreiras capilares. Rio de Janeiro, 2009. 86p. Dissertação de Mestrado - Departamento de Engenharia Civil, Pontifícia Universidade Católica do Rio de Janeiro.

Esta pesquisa esta orientada a avaliar o potencial de uso de um resíduo da indústria da fabrica de papel, localizada no estado de Rio de Janeiro, em sistemas de barreiras capilares para aterros sanitários. A utilização do resíduo da indústria de papel (RIP) trace um beneficio ambiental e econômico, pois na atualidade os materiais comumente usados como cobertores têm elevados custos. Neste estudo se realizam uma caracterização do RIP e uma simulação numérica usando o programa VADOSE/W 2007. O RIP é constituído por uma parte mineral e outra de fibra orgânica. A parte mineral é aproximadamente $70 \%$, composto principalmente de calcita e aragonita, atuando como cimento e cobrindo quase por completo as fibras. O RIP tem um elevado teor de umidade inicial e uma elevada contração sim apresentar trincas no processo de secagem. Ensaios de laboratório mostram valores de permeabilidade saturada na ordem de $10^{-8} \mathrm{~m} / \mathrm{s}$ para amostras no lado úmido da curva de compactação. Ensaios de curva de retenção de umidade usando a técnica de papel de filtro mostram uma diferença substancial em relação a outros RIPs encontrados na literatura pelo elevado valor de entrada de ar da ordem de 1000kPa. Simulações numéricas baseadas nas propriedades não saturadas do RIP mostram que ele pode ser utilizado como material fino de uma barreira capilar; sendo o contraste de permeabilidades não saturadas e sucção inicial chaves na seleção do material grosso. O programa VADOSE/W 2007 constitui uma ferramenta útil para á avaliação do funcionamento de sistemas de barreiras capilares onde se incluem condições climáticas.

\section{Palavras-chave}

Resíduo da indústria de papel. Caracterização de resíduos. Sistemas de barreiras capilares. Cobertura final. Aterro sanitário. 


\section{Abstract}

Bizarreta, Julio César Ortega; de Campos, Tácio Mauro Pereira. Evaluation of the potential use of waste paper sludge in capillary barrier systems. Rio de Janeiro, 2009. 86p. Dissertação de Mestrado Departamento de Engenharia Civil, Pontifícia Universidade Católica do Rio de Janeiro.

This research is oriented to evaluate the potential use of a waste industry manufactures of paper, in the state of Rio de Janeiro for capillary barrier systems for sanitary landfills. The use of waste paper industry (WPI) brings an environmental and economic benefit, because in actuality the cover materials have high costs. In this study, is made a characterization and a numerical simulation of WPI using the program VADOSE/W 2007. WPI is composed of one part mineral and other organic fiber. The mineral part is about $70 \%$, composed mainly of calcite and aragonite, acting as cement and covering almost completely the fibers. RIP has high initial moisture content and high contraction but does not have cracks in the drying process. Laboratory tests show values of saturated permeability in the order of $10^{-8} \mathrm{~m} / \mathrm{s}$ for samples in the wet side of compaction curve. Testing curve moisture retention using the filter paper method shows a substantial difference compared to other WPIs in the literature by the high Air Entry Value (AEV) of the order of $1000 \mathrm{kPa}$. Numerical simulations based on the properties of saturated RIP show that it can be used as fine material for a capillary barrier, and the contrast of unsaturated permeability and suction initial key in the material selection. Numerical simulations based on unsaturated properties of WPI showed that it can be used as material for a capillary barrier, and was verified that the contrast of unsaturated permeability and suction initial are key in the material selection, and was verified that the program VADOSE/W 2007 is a useful tool for evaluating the operation of capillary barrier systems which include climatic conditions.

\section{Palavras-chave}

Resíduo da indústria de papel. Caracterização de resíduos. Sistemas de barreiras capilares. Cobertura final. Aterro sanitário. 


\section{Sumário}

1 Introdução 1

1.1. Relevância e justificativa da pesquisa

1.2. Objetivo 2

1.3. Limitações da pesquisa 2

1.4. Organização da pesquisa 2

2 Composição mineralógica, estrutura morfológica e características químicas 4

2.1. Introdução 4

2.2. Revisão bibliográfica 4

2.3. Materiais e métodos 5

2.3.1. Composição mineralógica 5

2.3.2. Caracterização química 6

2.3.3. Caracterização morfológica 6

2.4. Resultados e discussão

2.4.1. Composição mineralógica

2.4.2. Características químicas 10

2.4.3. Estrutura morfológica 11

3 Caracterização física, compactação e contração 20

3.1. Introdução 20

3.2. Revisão bibliográfica 20

3.3. Materiais e métodos 22

3.4. Resultados e discussão 23

3.4.1. Caracterização física 23

3.4.2. Compactação 25

3.4.3. Contração 28

4 Curva de retenção 32

4.1. Introdução 32

4.2. Revisão bibliográfica 32

4.3. Materiais e métodos 33

4.4. Resultados e discussão 35 
4.4.1. Verificação da curva de calibração 35

4.4.2. Observações durante o ensaio 36

4.4.3. Resultados da curva de retenção usando a técnica de papel de filtro $\quad 40$

4.4.4. Modelagem da curva de retenção 41

4.4.5. Sucção, teor de umidade e índice de vazios 43

4.4.6. Comparação da curva de retenção com o RIP de Parent (2006) 45

5 Função de permeabilidade $\quad 46$

5.1. Introdução 46

5.2. Revisão bibliográfica 46

5.3. Materiais e métodos $\quad 47$

5.4. Resultados e discussão 48

5.4.1. Permeabilidade Saturada 48

5.4.2. Estimativa da função de permeabilidade 52

6 Análise numérica de sistemas de barreiras capilares 54

6.1. Introdução 54

6.2. Revisão bibliográfica 54

6.3. Análise numérica 56

6.3.1. Geometria do modelo 56

6.3.2. Condições ambientais $\quad 58$

6.3.3. Materiais $\quad 59$

6.3.4. Condições de contorno 61

6.4. Resultados e discussão da análise numérica 62

6.4.1. Análise 1

6.4.1.1. Simulação 1: areia SP sem infiltração por 180 dias 63

6.4.1.2. Simulação 2: areia SP com infiltração constante por 180 dias 65

6.4.1.3. Simulação 3: areia SP com infiltração variável 1 por 180 dias 66

6.4.1.4. Simulação 4: areia SP com infiltração variável 2 por 180 dias 68

6.4.1.5. Simulação 5: material RDC com infiltração constante por 180 dias 69

6.4.2. Análise $2 \quad 71$

6.4.2.1. Simulação 1: material SP sem infiltração na superfície em 90 dias 71

6.4.2.2. Simulação 2: material SP para taxas de infiltração (q)

de 0,5 e 2,05 mm/dia em 90 dias 72

6.4.2.3. Simulação 3: material SP para condições ambientais em 180 dias 74

6.4.2.4. Simulação 4: material RDC para condições ambientais em 180 dias 77

6.4.3. Comparação das simulações 78 
6.4.3.1. Simulações da análise $1 \quad 78$

6.4.3.2. Simulações da análise $2 \quad 80$

7 Conclusões e sugestões $\quad 82$

7.1. Conclusões

7.1.1. Composição mineralógica, estrutura morfológica e características químicas. $\quad 82$

7.1.2. Caracterização física, compactação e contração 82

7.1.3. Curva de retenção 83

7.1.4. Permeabilidade saturada $\quad 83$

7.1.5. Análise numérica de sistemas de barreiras capilares 84

7.2. Sugestões para trabalhos futuros $\quad 84$

$\begin{array}{lr}\text { Referencias Bibliográficas } & 86\end{array}$ 


\section{Lista de figuras}

Figura 2.1 - Procedimento de preparação das amostras para o MEV. $\quad 7$

Figura 2.2 - Espectro de difração de raios-X da amostra M3.

Figura 2.3 - Imagem de 50X de aumento em corte horizontal (a) e de corte vertical (b).

Figura 2.4 - Imagem com aumento de 200X de corte horizontal (a) e de corte vertical (b).

Figura 2.5 - Imagem com aumento de 500X de corte horizontal (a) e de corte vertical (b).

Figura 2.6 - Imagem com aumento de 2000X de corte horizontal (a) e vertical (b).

Figura 2.7 - Detalhe de uma fibra livre com um aumento de 3000X.

Figura 2.8 - Espectro médio de EDS tomada de modo geral da área da imagem com um aumento de 2000X.

Figura 2.9 - Espectros de EDS tomados nos pontos específicos na imagem de 3000X.

Figura 2.10 - Detalhe da imagem com aumento de 500X com a técnica de BSE em coloração de cinzas.

Figura 2.11 - Imagens, (a) 150X (Cabral et al. 2000), (b) 200X (Iberio 2007) e (c) 200X (Neste trabalho).

Figura 2.12 - Imagens, (a) 450X (Teixeira 2001) e (b) 500X (Neste trabalho). 18 Figura 2.13 - Imagens, (a) 2000X (Teixeira 2001) e (b) 3000X (Neste trabalho).19 Figura 3.1 Diferença do RIP retido e passante da peneira 200. Figura 3.2 Curva granulométrica completa. Peneiramento via lavagem 25 Figura 3.3 Resultado da curva de compactação pelo método de Proctor Normal do RIP

Figura 3.4 - Diferença das estruturas superficiais do RIP compactado a distintas umidades.

Figura 3.5 - Diferença das estruturas do RIP compactado a distintas umidades.27 Figura 3.6 - Comparação da curva de Proctor Normal do RIP com a literatura. 27 Figura 3.7 - Curvas de secagem dos espécimes.

Figura 3.8 - Variação da deformação volumétrica unitária com o teor de umidade de compactação.

Figura 3.9 - Espécimes ensaiados, após a secagem na estufa. 
Figura 3.10 - Contração da amostra compactado no mesmo molde do Proctor Normal.

Figura 3.11 - Variação de peso específico seco com o teor de umidade no processo de secagem.

Figura 3.12 - Variação de índice de vazios com o teor de umidade no processo de secagem.

Figura 4.1 - Armazenagem das amostras de RIP na técnica de papel de filtro. 34 Figura 4.2 - Sucção do papel de filtro estimada pela umidade do papel no dessecador

Figura 4.3 - Redução da área de contato do papel de filtro devido à contração do resíduo.

Figura 4.4 - Arqueamento da superfície pela contração e o contato do papel de filtro.

Figura 4.5 - Ruptura do espécime pelas forças de tração geradas nas bordas $\mathrm{RIP} / \mathrm{molde}$.

Figura 4.6 - Fungos após selagem por quatro dias da amostra compactada a $125 \%$ de umidade.

Figura 4.7 - Após a secagem na estufa, apresentam-se alguns rasgos da colônia de fungos no papel filtro.

Figura 4.8 - Curva de retenção dos primeiros espécimes de 125\% e 130\% de umidade.

Figura 4.9 - Curvas de retenção de secagem (S) e umedecimento (U).

Figura 4.10 - Estimativa da curva de retenção pelo modelo de Fredlund e Xing (1994).

Figura 4.11 - Estimativa da curva de retenção pelo modelo de van Genuchten (1980).

Figura 4.12 - Estimativa de curvas de retenção para diferentes umidades e índices de vazios iniciais

Figura 4.13 - Curvas de retenção de secagem, sucção por teor de umidade. 44

Figura 4.14 - Curva de secagem, teor de umidade por índice de vazios.

Figura 4.15 - Comparação da curva de retenção com a obtida por Parent (2006).

Figura 5.1 - Permeabilidade saturada para diferentes umidades de compactação com Proctor Normal.

Figura 5.2 - Imagens de lupa binocular de amostras com diferente umidade de compactação. 
Figura 5.3 Morfologia de amostras moldadas a diferentes umidades (a:195\% e

b:72\%)

Figura 5.4 - Variações de volume no ensaio de permeabilidade: a) recalque b) expansão

Figura 5.5 - Função de permeabilidade para a umidade de compactação de $76 \%$

Figura 5.6 - Função de permeabilidade para a umidade de compactação de $125 \%$

Figura 5.7 - Função de permeabilidade para a umidade de compactação de $162 \%$.

Figura 6.1 Esquema da máxima largura de desvio (Ld) e o ponto DDL

Figura 6.2 Esquema do aterro, condições de contorno e malhas de elementos finitos.

Figura 6.3 Partes da cobertura estudada $\quad 58$

Figura 6.4 Dados climáticos da estação do Galeão (Portocarrero, 2009) 58

Figura 6.5 Curvas de retenção dos materiais utilizados nas simulações. $\quad 60$

Figura 6.6 Curvas de função de permeabilidade de materiais utilizados nas simulações.

Figura 6.8 Vetores de velocidade e saturação aos 80dias usando o SP

Figura 6.9 Resultados da modelagem para o material SP com infiltração constante de $10^{-9} \mathrm{~m} / \mathrm{s}$.

Figura 6.10 Resultados da modelagem para o material SP com infiltração variável 1 (análise 1).

Figura 6.11 Resultados da modelagem para o material SP com infiltração variável 2 (análise 1).

Figura 6.12 Vetores de velocidade e saturação aos 30dias usando o RDC.

Figura 6.13 Resultados da modelagem para o material RDC com infiltração constante de $10^{-8} \mathrm{~m} / \mathrm{s}$.

Figuras 6.14 Perfis de sucção. (a) Topo X=5m e (b) Base X=29m. 71

Figura 6.15 Sucção na interface RIP/SP sem infiltração na superfície. 72

Figura 6.16 Fluxo ascendente de água pela interface RIP/SP.

Figuras 6.17 Percolação da água pela interface RIP/SP. (a) 0,5mm/dia

(b) $2,05 \mathrm{~mm} / \mathrm{dia}$

Figuras 6.18 Sucção na interfase do RIP/SP. (a) 0,5mm/dia (b) 2,205 mm/dia 73

Figuras 6.19 Barreira capilar com material SP no tempo de 30 dias $(q=0,5$ $\mathrm{mm} / \mathrm{dia})$. 
Figuras 6.20 Percolação da água pela interface RIP/SP pela

posição horizontal X. 75

Figuras 6.21 Sucção na interfase do RIP/SP pela posição horizontal X. $\quad 75$

Figuras 6.22 Perfil de saturação da cobertura na posição

X=29m (topo do talude).

Figuras 6.23 Perfil de saturação da cobertura na posição

$\mathrm{X}=5 \mathrm{~m}$ (base do talude). 76

Figuras 6.24 Percolação da água pela interface RIP/RDC. 77

Figuras 6.25 Sucção na interfase do RIP/RDC pela posição horizontal X. $\quad 77$

Figuras 6.26 Comparação das simulações da análise 1.

Figura 6.27 Variação das sucções no topo da camada SP para infiltração constante de $10^{-9} \mathrm{~m} / \mathrm{s} . \quad 79$

Figura 6.28 Variação das sucções no topo da camada RDC para infiltração constante de $10^{-9} \mathrm{~m} / \mathrm{s}$.

Figuras 6.29 Comparação das simulações variando a taxa de infiltração. $\quad 81$ 


\section{Lista de tabelas}

Tabela 2.1 - Composição mineralógica de RIP segundo a literatura revisada 4 Tabela 2.2 - Resultados da análise de fluorescência de raios-X da amostra M1. 8 Tabela 2.3 - Resultados da análise de fluorescência de raios-X da amostra M2 (550 ${ }^{\circ} \mathrm{C}$ de queima).

Tabela 2.4 - Resultados de análise quantitativa de raios-X para diferentes preparações de amostras.

Tabela 2.5 - Composição da amostra de RIP. 10

Tabela 2.6 - Comparação de pH com valores da literatura. 11

Tabela 2.7 - Resultados das características químicas analisadas. 11

Tabela 2.8 - Comparação das características do conjunto mineral-fibra com $\begin{array}{ll}\text { outros RIPs. } & 17\end{array}$

Tabela 3.1 - Resultados da caracterização física do RIP 23

Tabela 4.1 - Determinação da curva de retenção do RIP segundo diferentes autores. 33

Tabela 4.2 - Teor de umidade para $800 \mathrm{kPa}$ de sucção. 43

Tabela 4.3 - Comparação de VEA com a variação dos índices de vazios. $\quad 45$

Tabela 5.1 - Características morfológicas de três amostras de diferente umidade.5 Tabela 5.2 - Permeabilidade saturada e parâmetros da curva de retenção para diferentes umidades. 52

Tabela 6.1 Parâmetros e propriedades dos materiais da cobertura. $\quad 59$ 


\section{Lista de símbolos}

$\begin{array}{ll}e & \text { Índice de vazios } \\ e_{o} & \text { Índice de vazios inicial } \\ G_{s} & \text { Densidade dos grãos } \\ \mathrm{k}_{\text {sat }} & \text { Permeabilidade saturada } \\ \theta & \text { Teor de umidade volumétrico } \\ \theta_{\text {sat }} & \text { Teor de umidade volumétrico saturado } \\ \mathrm{S} & \text { Grau de saturação } \\ \mathrm{w} & \text { Teor de umidade gravimétrico } \\ \mathrm{w}_{\mathrm{o}} & \text { Teor de umidade gravimétrico inicial } \\ \mathrm{w}_{\mathrm{ot}} & \text { Teor de umidade ótima } \\ \mathrm{w}_{\mathrm{L}} & \text { limite de liquidez } \\ \mathrm{w}_{\mathrm{P}} & \text { limite de plasticidade } \\ \gamma_{d} & \text { Peso específico seco } \\ \gamma_{\mathrm{w}} & \text { Peso específico da água } \\ \mathrm{MO} & \text { Teor de matéria orgânica } \\ \mathrm{VEA} & \text { Valor de entrada de ar } \\ \mathrm{L}_{d} & \text { Máxima largura de desvio } \\ \mathrm{DDL} & \text { Ponto de começo da zona de transição } \\ \mathrm{q} & \text { Taxa de infiltração }\end{array}$

